\title{
Revisión
}

\section{Implicaciones metabólicas del consumo excesivo de fructosa}

\author{
Viviana Esquivel-Solís, Georgina Gómez-Salas
}

\section{Resumen}

En los últimos años se ha dado un auge importante en la producción de alimentos endulzados con edulcorantes distintos a la sacarosa o azúcar de mesa, que por aportar menos calorías, son utilizados ya sea para perder peso o para mantener un peso saludable, o bien, para ofrecer opciones alimenticias a individuos que padecen de diabetes o intolerancia a la glucosa. Uno de los edulcorantes de mayor utilización es el jarabe de maíz alto en fructosa, el cual, en comparación con la sacarosa, tiene un costo más bajo y un menor efecto sobre las concentraciones sanguíneas de glucosa.

Sin embargo, nuevas investigaciones están relacionando el consumo excesivo del jarabe de maíz alto en fructosa con la incidencia y prevalencia de enfermedades crónicas como la obesidad, la diabetes, las dislipidemias, el síndrome metabólico y las enfermedades cardiovasculares.

El presente artículo describe las posibles implicaciones metabólicas asociadas a enfermedades asociados con consumo excesivo de fructosa.

Descriptores: Dietas excesivas en fructosa, sirope de maíz alto en fructosa, obesidad, insulino resistencia y dislipidemia

Key words: fructose, corn syrup, high infructose, obesity, insulin resistance, dyslipidemia

Recibido: 23 de octubre de 2007

Aceptado: 31 de agosto de 2007

La fructosa es un edulcorante natural que fue introducido como un sustituto del azúcar de mesa a mediados de los años 70. Inicialmente, entre las ventajas del uso de la fructosa, se destacó el hecho de no ejercer un efecto significativo sobre la glicemia (con un índice glicémico de 20 en comparación con un índice glicémico de 100 para la glucosa) o la insulinemia, así tampoco sobre la producción de ácidos grasos y triglicéridos. ${ }^{1}$ A partir de esta y otras consideraciones, la fructosa fue incorporada en la elaboración de productos alimenticios dirigidos específicamente al tratamiento nutricional del paciente diabético. Sin embargo, en los últimos años se ha reconocido que el consumo de cantidades de fructosa que aporten un $25 \%$ ó más de la ingesta diaria de calorías, puede ocasionar desórdenes en el metabolismo de los lípidos y síntomas gastrointestinales. ${ }^{2,3}$ La siguiente revisión bibliográfica tiene como objetivo describir los

Escuela de Nutrición, Departamento de Bioquímica, Escuela de Medicina,Universidad de Costa Rica

Correspondencia:

Viviana Esquivel Solís. vesquive@nutricion.ucr.ac.cr

Georgina Gómez Salas. ggomez@cariari.ucr.ac.cr

ISSN 0001-6002/2007/49/4/198-202 Acta Médica Costarricense, $(02007$ Colegio de Médicos y Cirujanos hallazgos que relacionan el consumo excesivo de sirope de maíz alto en fructosa con el desarrollo de resistencia a la insulina, diabetes, obesidad y enfermedad cardiovascular.

\section{Digestión, absorción y metabolismo de la fructosa}

La principal fuente de fructosa es la sacarosa o azúcar de mesa, que proviene en general de la caña de azúcar o de la remolacha azucarera. Otras fuentes de fructosa son la miel de abeja y las frutas como: dátiles, higos, manzanas, uvas, fresas y moras. En el jugo de manzana, por ejemplo, el $65 \%$ de la energía proviene de la fructosa, y en el jugo de naranja, el $40-45 \%$, mientras que un $40 \%$ de los carbohidratos de la miel de abeja son moléculas de fructosa. ${ }^{3}$ 
La fructosa se utiliza como edulcorante en alimentos preparados, o se vende granulada para uso de los consumidores, a quienes erróneamente se les ha hecho creer que por no requerir de insulina para ser metabolizada, se puede utilizar sin restricción alguna. ${ }^{4}$

La principal fuente de fructosa a nivel de la industria de alimentos es el jarabe o sirope de maíz alto en fructosa, que se adiciona en gran cantidad de alimentos como cereales de desayuno, postres, repostería, helados, confites, jugos, bebidas azucaradas y refrescos gaseosos. ${ }^{4-6}$

El jarabe de maíz alto en fructosa es elaborado a partir de almidón de maíz, el cual es hidrolizado enzimáticamente hasta obtener moléculas de glucosa libre, que son posteriormente convertidas en moléculas de fructosa por medio de la enzima glucosa isomerasa. El jarabe de maíz alto en fructosa se clasifica de acuerdo con el contenido de fructosa en la mezcla $(42 \%, 55 \%$ ó $100 \%){ }^{3}$

Se ha estimado que el consumo de jarabe de maíz alto en fructosa en los Estados Unidos ha aumentado de forma alarmante en las últimas décadas, pasando de 230 gramos anuales per cápita en 1970, a 28.7 kilos por año en $1997 .{ }^{7}$ Algunos segmentos de la población estadounidense pueden llegar a consumir en promedio hasta 100 gramos de fructosa por día, proveniente de su utilización como edulcorante. ${ }^{3}$ De la misma manera, Basciano y colaboradores ${ }^{2}$ reportan que una persona puede consumir de 16-20 gramos de fructosa por día, proveniente de las frutas frescas, pero, el uso de fructosa como aditivo o edulcorante ha llegado a incrementar el consumo hasta aproximadamente 85-100 gramos diarios.

La sacarosa es hidrolizada a nivel intestinal por la enzima sacarasa en los monosacáridos constituyentes: fructosa y glucosa. La fructosa libre, consumida como tal o como producto de la hidrólisis enzimática de la sacarosa es absorbida principalmente en el duodeno y el yeyuno. ${ }^{6,8}$

Al igual que otros monosacáridos, la fructosa es absorbida por difusión facilitada a nivel de yeyuno, a través de una proteína transportadora denominada GLUT 5, en un proceso no dependiente de sodio. ${ }^{9-11}$

Durante el consumo excesivo y sostenido de fructosa, la tasa de absorción aumenta, lo que sugiere una adaptación fisiológica ante el incremento en la ingesta dietética. ${ }^{3,12}$

Una vez absorbida, la fructosa es transportada al hígado, donde es fosforilada por la enzima fructoquinasa a fructosa 1 fosfato, que se convierte en gliceraldehido 3 fosfato o dihidroxiacetona fosfato (Figura 1). Estas triosas fosfato podrán entrar en la vía glicolítica hasta formar piruvato y oxidarse posteriormente a Acetil CoA. ${ }^{8,13-14}$

En el hígado el Acetil CoA proporciona carbonos para la síntesis de ácidos grasos, triglicéridos y colesterol. Es por esta razón que al consumir grandes cantidades de fructosa se estimulan las vías glicolíticas y lipogénicas en la célula hepática, lo cual se ve favorecido por el hecho de que, contrario a la glucosa, la molécula de fructosa entra a la vía glicolítica sin pasar por el punto de regulación: la reacción catalizada por la fosfofructoquinasa. ${ }^{1}$ Esta enzima es inhibida alostéricamente por el citrato y el ATP, regulando, por lo tanto, la subsecuente producción de triglicéridos. ${ }^{9,12,15}$

Como se mencionó anteriormente, la molécula de Acetil CoA provee los carbonos necesarios para la síntesis de novo de ácidos grasos de cadena larga, que posteriormente son esterificados para formar triglicéridos., ${ }^{3,15}$ En animales de experimentación, como ratones, perros y primates, es conocido que dietas altas en energía a partir de fructosa o sacarosa inducen a la hiperlipidemia e hipertensión, lo cual eventualmente se asociaría con aumento del riesgo de enfermedades cardiovasculares. ${ }^{2-3,16}$

En el estudio realizado por Teff y colaboradores, ${ }^{17}$ se observó que en aquellas mujeres jóvenes que ingirieron bebidas endulzadas con fructosa, la concentración plasmática de triglicéridos incrementó más rápidamente y alcanzó una mayor concentración, que en aquellas que recibieron bebidas endulzadas con glucosa. Según Havel, ${ }^{3}$ existen reportes de que durante el consumo de fructosa se incrementa la síntesis de Apo B que antecede al aumento de las VLDL, lo cual, eventualmente, podría provocar un aumento de los triglicéridos. Se sabe que cambios en las VLDL son asociados a cambios en otras lipoproteínas como las HDL y las LDL pequeñas y densas, lo que podría relacionarse con el desarrollo de aterosclerosis. De igual manera, se ha propuesto que el aumento en la concentración sanguínea de triglicéridos, como consecuencia del consumo de altas cantidades de fructosa, se debe no solo a una mayor secreción de lipoproteínas de muy baja densidad, sino también a un menor aclaramiento de estas partículas por parte de las células de los tejidos periféricos, debido probablemente a una menor actividad enzimática de la lipasa lipoproteica.,

Por otro lado, en el estudio realizado por Kelley y Azhar, ${ }^{18}$ se propone que este aumento en los triglicéridos sanguíneos puede deberse al efecto sobre la acción del

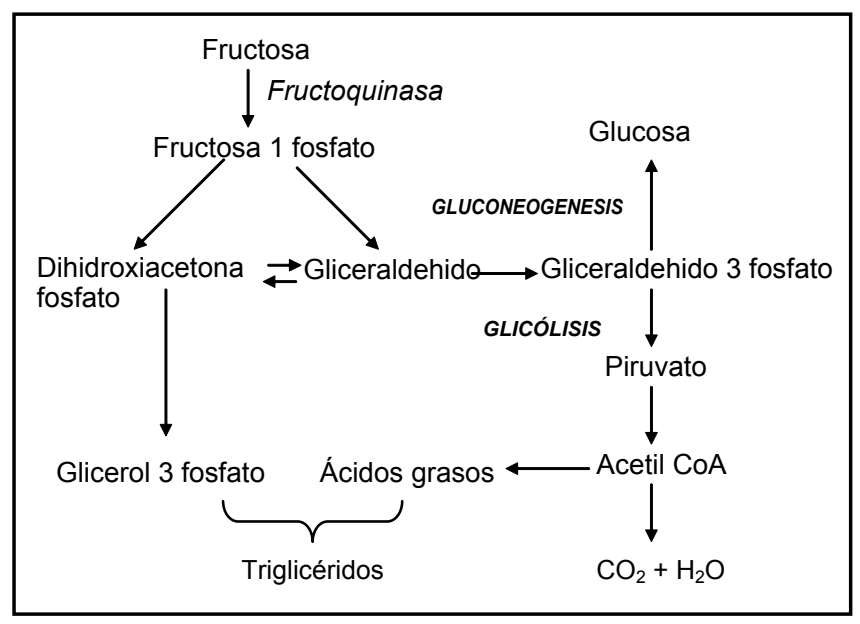

Figura 1. Metabolismo de la fructosa 
receptor PPR $\alpha$ observado en animales de laboratorio luego del consumo elevado de fructosa. La activación de este receptor estimula la expresión de genes relacionados con la oxidación de ácidos grasos y el metabolismo de las lipoproteínas. Los resultados del estudio demostraron que la fructosa puede disminuir la expresión hepática del receptor PPR $\alpha$ y los niveles de ARN mensajero de este receptor.

En otro estudio realizado por estos mismos autores se observó que las dietas altas en fructosa podrían estar alterando el metabolismo de los lípidos a través de la producción de citoquinas proinflamatorias, y que estos efectos podrían ser revertidos con la administración de inhibidores de las enzimas que participan en la síntesis de estas proteínas. ${ }^{18}$

No todos los estudios en los que se somete a individuos sanos al consumo excesivo de fructosa demuestran un efecto sobre los triglicéridos sanguíneos. Bantle y colaboradores, ${ }^{5}$ por ejemplo, observaron resultados similares a los anteriores en varones, pero no en mujeres, y no lograron demostrar cambios significativos en las concentraciones sanguíneas de colesterol.

\section{Resistencia a la insulina y Diabetes Mellitus}

La incidencia de diabetes tipo 2 ha aumentado a nivel mundial en las últimas décadas. La dieta es uno de los factores ambientales más influyentes en el desarrollo de esta enfermedad. Entre los componentes de la alimentación, el incremento en el consumo de fructosa se ha correlacionado con la ganancia de peso, el síndrome metabólico y la diabetes mellitus. ${ }^{19,20,21}$

Pequeñas o moderadas cantidades de fructosa parecen no tener impacto en el control glicémico del paciente con diabetes mellitus tipo 2. ${ }^{22,23}$ Pero, como se ha mencionado, el consumo excesivo podría empeorar problemas de hipertrigliceridemia o sobrepeso. ${ }^{3,24}$

La insulina se ha visto como una hormona anabólica que favorece la disposición de los nutrientes ingeridos para su empleo en los diferentes órganos, mediante la estimulación de la captura de glucosa por parte del músculo esquelético y el tejido adiposo, y la disminución de la glucogenólisis y gluconeogénesis hepáticas, así como la supresión de la lipólisis y la liberación de ácidos grasos libres de tejido adiposo. ${ }^{25}$

Los estudios realizados en pacientes diabéticos y normoglicémicos han demostrado que la fructosa produce un leve incremento de la glicemia y de la insulinemia postprandial, en comparación con otros carbohidratos. ${ }^{5} \mathrm{Sin}$ embargo, la recomendación del uso de este edulcorante por parte de pacientes diabéticos sigue siendo controversial, dado que como se indicó, el consumo excesivo de fructosa se ha asociado con aumento del peso corporal y con disminución de la insulina y la leptina, lo cual reduce la sensibilidad a la insulina. $5,26,27,28$
Daly $^{29}$ demostró, en estudios realizados en roedores, que las dietas altas en sacarosa y en fructosa reducen la sensibilidad a la insulina, pero sostienen la importancia de no descartar otros factores dietarios. También se ha observado en otros estudios con animales de laboratorio, que estas dietas incrementan el consumo total de energía, el peso corporal y la adiposidad. ${ }^{9,14,30}$

Dirlewanger, Schineiter, Jéquier, y Tappy ${ }^{14}$ proponen que la infusión aguda con fructosa induce a la insulino resistencia a nivel hepático y extrahepático, al aumentar en la síntesis intrahepática de Glucosa-6-fosfato.

Por otro lado, Wu y colaboradores ${ }^{31}$ evaluaron la asociación entre el consumo de fructosa, el índice glicémico, el consumo de carbohidratos y las concentraciones plasmáticas de péptido $\mathrm{C}$, que se ha asociado con resistencia a la insulina y con el desarrollo de diabetes tipo 2, y concluyeron que las dietas altas en fructosa y en índice glicémico se asocian con el aumento en la concentración del péptido $\mathrm{C}$, y por el contrario, el consumo de alimentos altos en fibra, como alimentos integrales, se vincula con la disminución de péptido C. Los autores sugieren que estos nutrientes desempeñan un papel divergente en el desarrollo de la insulino resistencia y la diabetes mellitus tipo 2 .

En un estudio citado por Basciano, Federico, y Adeli, ${ }^{2}$ en el que durante 28 días se brindó alimentación con fructosa, no se encontraron cambios en la concentración del receptor de insulina, pero sí se observó una disminución del $72 \%$ en la autofosforilación del receptor la insulina, tanto en el hígado como en el músculo.

\section{Fructosa y ganancia de peso}

La obesidad ha sido reconocida como una enfermedad metabólica que va más allá del desbalance entre el gasto y la ingesta de energía, y los estudios recientes han ampliado el conocimiento de la compleja interacción entre el papel regulador del adipocito, la composición de la dieta y el gasto energético. ${ }^{32}$

La prevalencia de la obesidad se ha incrementado de forma paralela al consumo de fructosa. ${ }^{5}$ Pero, existe poca información sobre el efecto de la fructosa sobre la secreción de reguladores endocrinos del balance energético como la insulina, la ghrelina y la leptina.

En el estudio realizado por Teff y colaboradores, ${ }^{17} \mathrm{se}$ observó que los sujetos recibieron comidas en las cuales el $30 \%$ de las calorías eran aportadas por una bebida endulzada, ya fuera con glucosa o con fructosa. Se presentaron diferentes respuestas metabólicas y hormonales. La respuesta glicémica e insulinémica fue un $66 \%$ y un $65 \%$ menor, respectivamente, en los sujetos que ingirieron la bebida con fructosa en comparación con los que ingirieron glucosa.

Bantle $^{5}$ propone que al producir un menor efecto insulinémico, la ingesta excesiva de fructosa podría a la vez 
estar aumentando el apetito y, por lo tanto, la ingesta de alimentos. El investigador basa sus apreciaciones en el hecho de que la insulina estimula la liberación de leptina por parte del adipocito, y dado que la leptina ejerce un efecto anorexígeno en el hipotálamo, los niveles inferiores de insulina y leptina, luego de la ingesta de fructosa, podrían ejercer un menor efecto inhibidor del apetito que la ingesta de otros carbohidratos. Sin embargo, Schorin ${ }^{6}$ asegura que el dramático aumento de la obesidad a nivel mundial se ha dado independientemente del incremento en el uso de siropes altos en fructosa.

Una de las mayores preocupaciones es el manejo de la información que poseen algunos profesionales de la salud, y aún más las personas que consideran que estos alimentos por ser "dietéticos" pueden consumirse libremente. La publicidad en medios de comunicación masivos y en "ferias de la salud", muchas veces por desconocimiento brinda información errónea a quienes en la mayoría de los casos buscan de manera desesperada alimentos que puedan consumirse sin restricción.

Es importante que la información descrita en este artículo sea fuente de inspiración para futuras investigaciones y que, eventualmente, se evalúen con mayor rigurosidad los efectos de productos artificiales adicionados a los alimentos o preparaciones, a fin de observar y prevenir las secuelas en el largo plazo.

Es de interés nacional que los costarricenses volvamos a consumir alimentos de todos los grupos (frutas, vegetales, cereales, leguminosas, lácteos, grasas, azúcares y carnes) en las cantidades adecuadas, y que más que buscar opciones de "libre consumo", moderemos y procuremos consejería para seguir dietas balanceadas y saludables, que promuevan una menor inversión de recursos económicos y una mejor calidad de vida.

La alimentación es uno de los componentes que se pueden modificar para lograr un estilo de vida saludable, por lo que la adecuada nutrición y el control del peso deben ser un objetivo fundamental para los consumidores y para la industria alimentaria.

\section{Abstract}

During recent years there has been a remarkable increase in the production of foods sweetened sugar substitutes with less caloric value, that are used in dietary programs to lose weight to maintain a healthy weight as well as to offer options for individuals with diabetes or glucose intolerance.

One of the most common sweeteners is high fructose corn syrup, a less expensive sweetener that has a lower postprandial hyperglycemic effect compared to sucrose.
However new research has shown that prolonged consumption of high fructose diet would contribute to the development of chronic diseases such as obesity, diabetes, lipid disorders, metabolic syndrome and cardiovascular diseases.

This paper describes the possible metabolic implications related to different diseases associated with an excessive fructose consumption.

\section{Referencias}

1. Mehnert H. 1976. Sugar substitutes in the dieabetic diet. Int Z Vitam Ernahrungsforsch Beih 15:295-324.

2. Basciano H, Federico L, Adeli K. 2005. Fructose, insulin resistance, and metabolic dislipidemia, Nutrition and Metabolism, 2:5.

3. Havel P. 2005. Dietary fructose: Implications for dysregulation of energy homeostasis and lipid/carbohydrate metabolism. Nutrition Review 63: 133-157

4. Hein GL, Storey M, White J, Lineback DR. 2005. Highs and Lows of High fructose corn syrup. A Report form the Center fro Food and Nutrition Policy and Its Ceres Workshop. Nutrition Today 40:253256

5. Bantle JP. 2006. Is fructose the optimal low glycemic index sweetener? Nestle Nutr Workshop Ser Clin Perform Progamme. 11:83-91

6. Schorin MD. 2005. High fructose corn syrups. Part 1 and 2. Nutrition Today. 40:248-252.

7. Putman J. 1999. Food Consumption, prices and expenditures, 19701991. In Economic Research Service Washington DC. US Department of Agricultu

8. Nelson DL, Cox MM. 2004. Lehninger principles of Biochemistry. $4^{\text {th }}$ Ed. New York: Worth Pusblishers

9. Bray G, Nielsen S, Popkin B. 2004. Consumption of high-fructose corn syrup in beverages may play the rol in the epidemic of obesity. Am J Clin Nut. 79:537-43

10. Fornaguera J. y Gómez G. 2004. Bioquímica: la ciencia de la vida-San José, Costa Rica, Editorial Universidad Estatal a Distancia, 340 p.

11. Benyon S. 1998. Metabolismo y nutrición. Edición en español Harcort Brace de España, S.S, España, 244 p.

12. Cui XL, Schlesier AM, Fisher EL, Cequeira C, Ferraris RP. 2005. Fructose- induced increases in neonatal rat intestinal fructose transport involve the PI3-kinase/Akt signalling pathway. Am J Physiol Gastrointest Liver Physiol 288:G1310-G1320

13. Bantle J, Raatz S, Thomas W, Goergopoulos A. 2000. Effects of dietary fructose on plasma in healthy subjects. Am J Clin Nutri 72:1128-34

14. Dirlewanger M, Schineiter P, Jéquier E, Tappy L. 2000. Effects of fructuose on hepatic glucose metabolism in humans. Am J Physiol Endocrinol Metab, 279:E907-E911

15. Elliott S, Kein N, Stern J, Teff K, Havel P. 2002. Fructuose, weight gain, and the insulin resistence syndrome. AM J Clin Nutr 76:91122

16. Kelley GL, Azhar S. 2005. Reversal of high dietary fructose-induced PPAR $\alpha$ suppression by oral administration of lipoxygenase cyclooxygenase inhibitors. Nutrition \& Metabolism 2:18

17. Teff KL, Elliot SS, Tschop M, Kieffer TJ, Rader D, Heiman M, Townsend RR, Keim NL, D'alessio D, Havel PJ. 2004. Dietary fructose reduces circulating insulin and leptin, attenuates postprandial suppression of ghrelin and increases triglycerides in women. J Clin Endocrinol Metab 89:2963-2972. 
18. Kelley GL, Allan G, Azhar S. 2004. High dietary fructose induces a hepatic stress response resulting in cholesterol and lipid dysregulation. Endocrinology 145:548-555.

19. Musick T, Cymet TC. 2006. Carbohydrate and calories: is not what we use to think. Compr Ther 32:47-50.

20. Sartorelli DS, Cardoso MA. 2006. Association between dietary carbohydrates and type 2 diabetes mellitus: epidemiological evidence. Arq Bras Endocrinol Metabol 50:415-26.

21. Rayssiguier Y, Gueux E, Nowacki W, Rock E, Mazur A. 2006. High fructose consumption combined with low dietary magnesium intake may increase the incidence of metabolic syndrome by inducing inflammation. Magnes Res 19:237-43.

22. Hawkins M, Grabriely I, Wozniak R, Vilcu C, Shamoon H, Rossetti L. 2002. Fructose improves the ability of hyperglycemia per se to regulates glucose production in type 2 diabetes. Diabetes 51:606614.

23. Ganon MC, Nutall FQ, Westphal SA, Fan S, Ercan-Fang N. 1998. Metabolic response to carbohydrate, high-starch meals compared with moderate carbohydrate low-starch meals in subjects with type 2 diabetes. Diabetes Care 21:1619-1626.

24. Gross LS, Li L, Ford ES, Liu S. 2004. Increased consumption of refined carbohydrate and the epidemic of type 2 diabetes in the United States : an ecologic assessment. Am J Clin Nutr 19:774-9.
25. Davail S, Ridea N, Bernardet MD, Guy G, Hoo-Paris R. 2005. Effect of dietary fructose on liver steatosis in overfed mule ducks. Horm Metab Res 37:32-35.

26. Bizeau ME, Pagliassotti MJ. 2005. Hepatic adaptation to sucrose and fructose. Metabolism Clinical and Experimental. 54:1189-1201.

27. Bessesen H. 2001. The role of Carbohydrates in Insuline Resistence. J. Nutr 131:2782S-2786S.

28. Zammit VA, Waterman IJ, Topping D, Mc Kay G. 2001. Insulin stimulation of hepatic triacylglicerol secretion and the etiology of insuline resistance. J. Nutr 131:2074-2077

29. Daly M. 2003. Sugars, insulin sensitivity, and the postprandial state. Am J Clin Nutric; 78 (suppl): 865S-72S.

30. Jürgens H, Haass W, Castañeda T, Schümann A, Koebnick C, Dombrowski F, Otto B, Nawrocki A, Schere P, Spranger J, Ristow M, Joost H, Havel P, Tschöp M. 2005. Consuming fructose-sweetened beverages increases body adiposity in mice. Obesity research 13: 1146-1156.

31. Wu T, Giovannucci E, Pischon T, Hankinson S, Nader J, Rimn E. 2004. Fructose, glycemic load, and quantity and quality of carbohydrate in relation to plasma C-peptide concentrations in US women. Am J Clin Nutr 80:1043-9.

32. Walker CG, Zariwala MG, Holness MJ, Sugden MC. 2007. Diet obesity and diabetes: a current update. Clin Sci 112:93-111. 